\title{
Rationale of Endoscopic Spine Surgery: A New Paradigm Shift In Spine Surgery From Patient's benefits to Public Interest In This New Era of Pandemic
}

\author{
Hyeun Sung Kim², Pang Hung $\mathrm{Wu}^{2}$, Il-Tae Jang ${ }^{1}$ \\ ${ }^{1}$ Department of Neurosurgery, Nanoori Gangnam Hospital, Seoul, Spine Surgery, Seoul, Republic of Korea \\ ${ }^{2}$ Department of Orthopaedic Surgery, National University Health System, Jurong Health Campus, Singapore
}

Corresponding Author:

Hyeun Sung Kim, MD, PhD

Department of Neurosurgery, Nanoor

Hospital Gangnam, Seoul 731, Eonju-ro, Gangnam-gu, Seoul, Republic of Korea

Tel: $+82-2-6003-9767$

Fax: +82-2-3445-9755

Email: neurospinekim@gmail.com

Received: February 03, 2021

Revised: February 22, 2021

Accepted: March 05, 2021
Advancement of technology and surgical skills act in synergy to lead to exploration of new solutions in spine surgery. One of the key areas of spine innovation is endoscopic spine surgery and its application to a broader spectrum of conditions with the aim of reducing perioperative morbidities, soft tissue and bony conservation and yet achieving long term target outcomes of gold standard traditional open spine surgery. Twenty first century marks the new century of opportunities and challenges, in the face of threat of Coronavirus pandemic and difficult circumstances in hospital bed management and limitation in medical resources, minimally invasiveness is evolving from individual patients' benefits to public interest.

Key Words: Endoscopic Spine Surgery, Uniportal Endoscopic Spine Surgery, Biportal Endoscopic Spine Surgery, Unilateral Biportral Endoscopy

\section{INTRODUCTION}

The year of 2020 as a dawn of a new century presented new challenges in the world of medicine. The emergence of Coronavirus-19 (COVID-19) had led to new perspective in the treatment of patients. Many countries went into various versions of locked down or limitation of social interactions restrictions order in different period of the year 2020, health care institutions and hospitals are overwhelmed with patients requiring medical attention for Coronavirus. Many chronic and degenerative surgical conditions treatment are placed on the lower priority in the hospital administration point of view. Elective spine surgeries are postponed or cancelled for sustained period of time. Day surgeries are preferred to surgeries requiring hospital stay unless it is an emergency. Inpatient beds are prioritized for treatment of patients who are in respiratory distress, reserve beds are also kept empty to preserve space in preparation for a spike in Coronavirus situation. As the countries are preparing themselves for the new normal, spine surgery has to keep up in innovation, technical development and protocol generation for the new era of preservation of hospital resources. Endoscopic Spine Surgery has undergone several generations of development and has replaced many of the open surgeries as a treatment for patient with degenerative spinal conditions in some of the surgeons' practice ${ }^{10)}$.
The key objectives of endoscopic spine surgery is favorable to the treatment of spinal conditions in this era of Coronavirus pandemic ${ }^{16)}$. They are minimizing pain and early mobilization, minimizing the number of personnel and surgical assistants, reducing resources on respiratory support equipment during anesthesia, reduce usage of intensive care unit and high dependency and yet achieving target clinical outcome with minimally invasive benefits. In this editorial we discuss the rationale of endoscopic spine surgery and how it has evolved from just benefiting the patients to public interest in this new era of pandemic. In this special endoscopic spine surgery edition, we collated a series of articles which provides perspective on how endoscopic spine surgery is a new paradigm shift for spine surgery practice especially in this era of pandemic (Figure 1).

\section{Minimize Pain and Early Mobilization}

The key feature for day surgery is minimizing postoperative pain, soft tissue preservation and small wound size for easy wound care. Lee et al showed there is decreased soft tissue and muscle injury in endoscopic as compared to microscopic surgery with low creatinine kinase as marker of soft tissue injury postoperatively ${ }^{11)}$. Similar findings were found in Heo et al showing promi sing better pain relief, preservation of soft tissue and achieve similar good clinical outcomes as microscopic surgery in biportal 


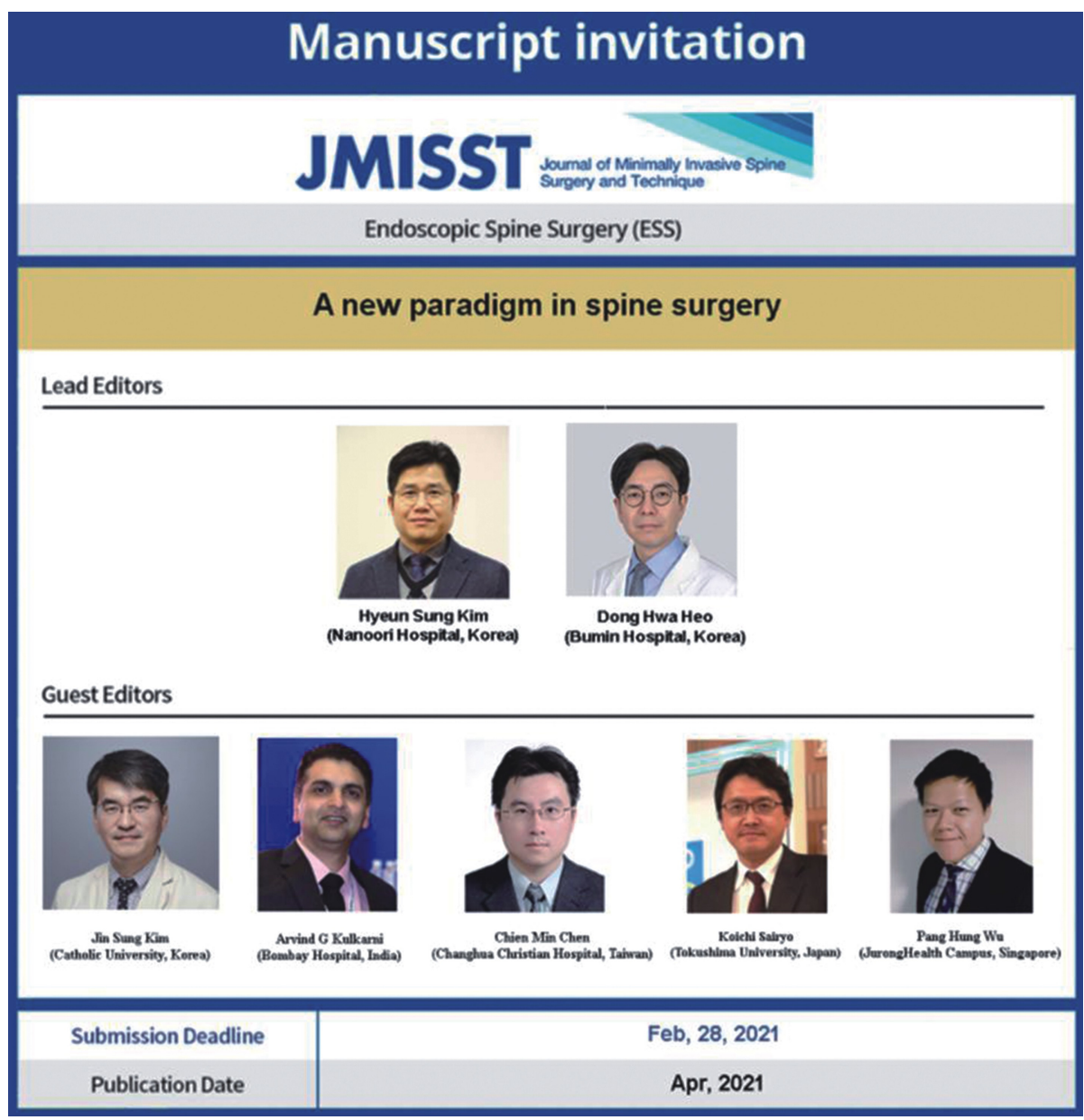

Figure 1. Editorial Team for journal of minimally invasive spine surgery and technique endoscopic spine surgery edition: A new paradigm in spine surgery.

and uniportal endoscopic surgery ${ }^{4)}$.

\section{Minimize Personnel in Operating Theatre}

In a typical case of uniportal and biportal and endoscopic surgery, the need for assistant surgeon is optional rather than essential. As there is only one incision in uniportal endoscopic surgery, there is no role in assistant surgeon in the operation as it is performed by a single surgeon only. In biportal surgery, although there are 2 incisions and some surgeons have a preference for a surgical assistant to assist in osteotomy and holding of retractor, the need for assistant is optional rather than essential. As there are working portal retractor designed for the purpose of keeping working portal opened. There are few trays of instruments required for either uniportal and biportal surgery, hence there is less demand on nursing staff as well. A typical of endoscopic spine surgery is performed with one operating surgeon, one scrubbed nurse, one radiographer, one circulating nurse and an anesthetist. Other operating personnel re optional. The advantage of decreasing the need for assistant surgeon and nursing support in endoscopic surgery: (1) decrease manpower cost as part of operational cost for hospital administration, (2) decrease cross infection risk among patient - healthcare workers and healthcare workers - healthcare workers during COVID-19 pandemic, (3) decrease wound infection risk and (4) privacy for patients.

Reduce Resources On Respiratory Support Equipment and Reduce Usage Of Intensive Care Unit And High Dependency

Many of the endoscopic procedures can be performed with local anesthesia and sedation ${ }^{7,22,23)}$, or as epidural anesthesia ${ }^{5}$, this decrease the need for respiratory support equipment used for endotracheal intubation for general anesthesia. During the critical period with high case load in COVID-19, there is shortage of protective personal equipment and respiratory support equipment. The ability to perform surgery without the use of general anesthesia and hence decreasing the possible need of intensive care unit and high dependency contributes in preservation of the supplies of these limited resources.

Reduce Hospital Inpatient Requirement: Demands For Day Surgery Procedures.

Recently some of the hospitals in the world has developed protocols in order to streamline the work processes in order 
to achieve high percentage of day surgeries their spine surgery practice. Wu et al highlighted that as a response for COVID-19 pandemic, their team set up a "Lumbar Endoscopic Awake Discectomy Surgery (LEADS)" protocol which consisted of 3 components: using moderate sedation and local anaesthesia to perform transforaminal endoscopic lumbar discectomy, same-day discharge for all patients who had undergone transforaminal endoscopic lumbar discectomy, and telemedicine communication at first week for follow-up with in-person visits 6 weeks after surgery. With the use of zoom and other tools for teleconsult/telemedicine, patients who had undergone endoscopic spine surgery can minimize visits to hospital. As the wound in his series are closed with tissue glues and no removal of stitches are required, many of the patients do not require physical consult and hence minimize risk of cross transmission of diseases and virus in hospital ${ }^{16)}$. While day surgery is not limited to endoscopic spine surgery alone, with typically one to two $1 \mathrm{~cm}$ scar in endoscopic spine surgery, less wound care related hospital visits would help in redu cing cost, providing convenience and optimization of social distancing in this time of pandemic.

\section{Target Clinical Outcome Achieved with Minimally Invasive Technology}

Several endoscopic surgeons have gradually broaden the indications for endoscopic spine surgeries and hence replacing a large portion of their open surgical load with endoscopic spine surgery ${ }^{2,10)}$. Comparison among microscopic tubular, uniportal and biportal endoscopic surgeries showed favorable perioperative outcomes, facet conservation and less muscular damages in endoscopic surgeries, ${ }^{4,111}$. There is increase in the benefit zone in endoscopic spine surgery to include more complex spinal procedures evolving from discectomy and decompression to fusion procedures ${ }^{1,3,17)}$. Posterior and anterior cervical decompression procedures have produced comparable good results with gold standard anterior cervical discectomy and fusion in some of the series published ${ }^{9,14,20,21)}$. Expansion of indication to spinal cord level degenerative pathologies in cervical and thoracic spine had led to potential decrease in perioperative morbidities of these high risk surgeries ${ }^{12,13,18)}$. Expansion of indications to include patients with mild scoliosis and narrow foramen involving endoscopic fusion has provided good clinical outcomes and achieve the benefits of minimally invasive surger ${ }^{6,19}$. Endoscopic fusion can be performed without general anesthesia with good clinical outcomes ${ }^{15}$. While we are closely monitoring the long term outcomes of these new endoscopic expansion of indications, the potential benefits of this branch of sub specialization of spine surgery is promising ${ }^{8,17)}$.

\section{CONCLUSION}

As part of medical fraternity, spine surgeons do our part in the fight against COVID-19, endoscopic spine surgery has inherent potential to be surgery of choice not only for patients' benefits but for public interests to decrease risk of spread of COVID-19, conservation of respiratory equipment and maintaining elective surgery service for patients who needed spine surgery during this era of pandemic and for the future.

\section{CONFLICT OF INTEREST}

Dr. HS Kim is an editorial board member of the journal but was not involved in the peer reviewer selection, evaluation, or decision process of this article. There are no other potential conflicts of interest relevant to this article to declare.

\section{REFERENCES}

1. Ahn Y: Endoscopic spine discectomy: Indications and outcomes. International Orthopaedics 43:909-916, 2019

2. Choi G, Pophale CS, Patel B, Uniyal P: Endoscopic Spine Surgery. Journal of Korean Neurosurgical Society 60:485-497, 2017

3. Hasan S, Hartl R, Hofstetter CP: The benefit zone of full-endoscopic spine surgery. J Spine Surg 5:S41-S56, 2019

4. Heo DH, Lee DC, Park CK: Comparative analysis of three types of minimally invasive decompressive surgery for lumbar central stenosis: Biportal endoscopy, uniportal endoscopy, and microsurgery. Neurosurg Focus 46:E9, 2019

5. Kang SY, Kashlan ON, Singh R, Rane R, Adsul NM, Jung SC, et al.: Advantages of the Combination of Conscious Sedation Epidural Anesthesia Under Fluoroscopy Guidance in Lumbar Spine Surgery. Journal of Pain Research 13:211-219, 2020

6. Kim H-S, Wu PH, Lee YJ, Kim DH, Jang IT: Technical Considerations of Uniportal Endoscopic Posterolateral Lumbar Interbody Fusion: A Review of Its Early Clinical Results in Application in Adult Degenerative Scoliosis. World Neurosurgery, 2020

7. Kim HS, Adsul N, Kapoor A, Choi SH, Kim JH, Kim KJ, et al.: A Mobile Outside-in Technique of Transforaminal Lumbar Endoscopy for Lumbar Disc Herniations. Journal of visualized experiments: JoVE, 2018

8. Kim HS, Wu PH, Jang I-T: Gurrent and Future of Endoscopic Spine Surgery: What are the Common Procedures we Have Now and What Lies Ahead? World Neurosurgery 140:642-653, 2020

9. Kim HS, Wu PH, Lee YJ, Kim DH, Kim JY, Lee JH, et al.: Safe Route for Cervical Approach: Partial Pediculotomy, Partial Vertebrotomy Approach For Posterior Endoscopic Cervical Foraminotomy and Discectomy. World Neurosurg 2020

10. Kim HS, Wu PH, Raorane HD, Jang IT: Generation Change of Practice in Spinal Surgery: Can Endoscopic Spine Surgery Expand its Indications to Fill in the Role of Conventional Open Spine Surgery in Most of Degenerative Spinal Diseases and Disc Herniations: A Study of 616 Spinal Cases 3 Years. Neurol India 68: 1157-1165, 2020

11. Lee C-W, Yoon K-J, Ha S-S: Comparative Analysis between Three Different Lumbar Decompression Techniques (Microscopic, Tubular, and Endoscopic) in Lumbar Canal and Lateral Recess Stenosis: Preliminary Report. BioMed Research International 2019:60784696078469, 2019

12. Lin Y, Rao S, Li Y, Zhao S, Chen B: Posterior percutaneous fullendoscopic cervical laminectomy and decompression for cervical stenosis with myelopathy: A technical note. World Neurosurg, 2019 
13. Ruetten S, Hahn P, Oezdemir S, Baraliakos X, Merk H, Godolias $\mathrm{G}$, et al.: Full-endoscopic uniportal decompression in disc herniations and stenosis of the thoracic spine using the interlaminar, extraforaminal, or transthoracic retropleural approach. Journal of Neurosurgery Spine 29:157-168, 2018

14. Ruetten S, Komp M, Merk H, Godolias G: Full-endoscopic cervical posterior foraminotomy for the operation of lateral disc herniations using 5.9-mm endoscopes: A prospective, randomized, controlled study. Spine (Phila Pa 1976) 33:940-948, 2008

15. Wang MY, Grossman J: Endoscopic minimally invasive transforaminal interbody fusion without general anesthesia: Initial clinical experience with 1-year follow-up. Neurosurgical focus 40:E13, 2016

16. Wu PH: Early Career Challenges in Setting Up an Endoscopic Spine Surgery Practice. World Neurosurgery 144:264-269, 2020

17. Wu PH, Kim HS, Jang I-T: A Narrative Review of Development of Full-Endoscopic Lumbar Spine Surgery. Neurospine 17:S20S33, 2020

18. Wu PH, Kim HS, Kim J-Y, Lee YJ, Kim DH, Lee JH, et al.: Uniportal thoracic endoscopic decompression using one block resection technique for thoracic ossified ligamentum flavum technical report. Interdisciplinary Neurosurgery 23:100963, 2021

19. Wu PH, Kim HS, Lee YJ, Kim DH, Lee JH, Jeon JB, et al.: Uni- portal Full Endoscopic Posterolateral Transforaminal Lumbar Interbody Fusion with Endoscopic Disc Drilling Preparation Technique for Symptomatic Foraminal Stenosis Secondary to Severe Collapsed Disc Space: A Clinical and Computer Tomographic Study with Technical Note. Brain Sci 10, 2020

20. Wu PH, Kim HS, Lee YJ, Kim DH, Lee JH, Yang K-H, et al.: Posterior endoscopic cervical foramiotomy and discectomy: Clinical and radiological computer tomography evaluation on the bony effect of decompression with 2 years follow-up. European Spine Journal, 2020

21. Yadav YR, Ratre S, Parihar V, Dubey A, Dubey MN: Endoscopic partial corpectomy using anterior decompression for cervical myelopathy. Neurol India 66:444-451, 2018

22. Yoshinari H, Tezuka F, Yamashita K, Manabe H, Hayashi F, Ishihama Y, et al.: Transforaminal full-endoscopic lumbar discectomy under local anesthesia in awake and aware conditions: The inside-out and outside-in techniques. Current Reviews in Musculos keletal Medicine 12:311-317, 2019

23. Zhu Y, Zhao Y, Fan G, Gu G, Sun S, Hu S, et al.: Comparison of the effects of local anesthesia and epidural anesthesia for percutaneous transforaminal endoscopic discectomy in elderly patients over 65 years old. International Journal of Surgery 48:260-263, 2017 